\title{
DNA Loss and Related Alterations in Long-term Cultures of Diploid, Tetraploid and Octaploid Meth-A Cells
}

\author{
Kohzaburo Fujikawa-Yamamoto*, Minoru Miyagoshi \\ and Hiroko Yamagishi \\ Division of Cell Medicine, Research Institute of Medical Science, Kanazawa Medical University, \\ Uchinada, Ishikawa 920-0293, Japan
}

Received July 25, 2005; accepted August 23, 2005

\begin{abstract}
Summary The DNA content of polyploid cells sometimes decreases during subculturing. The mechanism of DNA reduction is not yet known. Precise measurements of DNA decay and related alterations in polyploid cells will be required to understand the mechanism. Diploid, tetraploid and octaploid Meth-A cells were continuously cultured for $244 \mathrm{~d}$ and the cellular DNA content was measured from the DNA histograms. The DNA content decays gradually with day $\mathrm{t}$ as expressed by $\mathrm{f}(\mathrm{t})=\mathrm{I}_{\mathrm{p}} \exp \{-\mathrm{G}(\mathrm{t})\}$, where $\mathrm{I}_{\mathrm{p}}$ is the initial ploidy, $\mathrm{G}(\mathrm{t})=\mathrm{at} /\{\exp (-\mathrm{bt})+\mathrm{ct}\}$, and $\mathrm{a}, \mathrm{b}$ and $\mathrm{c}$ are the following parameters: $a=0.026, b=0.01$ and $c=0.0175$ (for tetraploid cells) or $c=0.01$ (for octaploid cells). The DNA content of diploid Meth-A cells was constant within the experimental errors. The DNA loss of polyploid cells was confirmed by a decrease in chromosome number. The cellular morphology changed in diploid and octaploid Meth-A cells, but not in tetraploid cells, suggesting that DNA loss and morphology alteration are independent. The doubling time shortened with culture time in the tetraploid and octaploid cells. The findings suggested that chromosomes are not independent in polyploid cells.
\end{abstract}

Key words DNA loss, Ploidy degradation, Tetraploid Meth-A cells, Octaploid Meth-A cells, Flow cytometry.

The DNA content of mammalian diploid cells is well preserved during subculturing; however, that of polyploid cells is not. The DNA content of polyploid cells sometimes decreases gradually, and occasionally it decreases abruptly by half. A few studies of DNA degradation in polyploid cells have been reported. Moor, et al. (1968) concluded that near triploid is the terminal ploidy of neartetraploid Ehrlich's ascites tumor cells based on 3 years of observation. Harris (1971) showed that the chromosome number was constant in diploid cells but decreased with subculturing in tetraploid and octaploid pig kidney cells. Graves and McMillan (1984) reported that tetraploid DON and BI Chinese hamster cells were karyotypically stable but the octaploid cells were not. In spite of these long-term studies, the mechanism of DNA content reduction is not yet known.

Meth-A cells always include a small population of large cells that are produced from diploid cells by spontaneous polyploidization and are eventually removed by apoptosis (Fujikawa-Yamamoto et al. 1997). Meth-A cells may be particularly susceptible to polyploid transformation. Tetraploid Meth-A cell lines have been reproducibly established from diploid Meth-A cells highly polyploidized by demecolcine (Fujikawa-Yamamoto et al. 2001). Octaploid Meth-A cells were also produced from tetraploid cells highly polyploidized by demecolcine (Fujikawa-Yamamoto et al. 2003). The diploid, tetraploid and octaploid Meth-A cells showed marked differences in the expression of cell surface hydrocarbon chains, suggesting that the ploidy transformation is accompanied by functional alterations (Fujikawa-Yamamoto and Sakuma 2003). The diploid, tetraploid and octaploid Meth-A cell lines may provide a cell system for investigating polyploid cells.

* Corresponding author, e-mail: fujikawa@kanazawa-med.ac.jp 
In this study, changes in DNA histograms, chromosome numbers, growth morphology and doubling time of diploid, tetraploid and octaploid Meth-A cells were measured for $244 \mathrm{~d}$ to elucidate the features of DNA loss in polyploid Meth-A cells. A possible explanation for the observations will be mentioned

Materials and methods

Cells

Meth-A cells (a methylcholanthrene-induced mouse abdominal dropsy sarcoma cell line) were maintained in a humidified atmosphere of $5 \% \mathrm{CO}_{2}$ at $37^{\circ} \mathrm{C}$ as suspension cultures in a Leibovitz's L15 : Ham's F10 mixture (7:3) supplemented with 10\% fetal bovine serum (CELLect GOLD, ICN Biomedicals, Aurora, OH, USA), streptomycin $(100 \mu \mathrm{g} / \mathrm{ml})$ and penicillin $(50 \mathrm{units} / \mathrm{ml})$. The tetraploid and octaploid Meth-A cells were cultured under the same conditions described above.

\section{Cell culturing for $244 d$}

Tetraploid and octaploid Meth-A cells stored at $-135^{\circ} \mathrm{C}$ in the early stage of establishment were thawed and cultured. Diploid Meth-A cells stored at $-135^{\circ} \mathrm{C}$ were also thawed and cultured. The diploid, tetraploid and octaploid Meth-A cells were subcultured almost every day by $1: 2$ or 1 : 4 dilution in culture flasks $\left(25 \mathrm{~cm}^{2}\right.$, Corning Costar Co., Acton, MA, USA). The cell number was counted using a hemocytometer at the time of subculturing. The cell density was maintained at more than $2 \times 10^{5}$ cells/flask. The DNA histograms of the cell populations were measured by flow cytometry (FCM). For controls, diploid, tetraploid and octaploid Meth-A cells stored at $-135^{\circ} \mathrm{C}$ were used immediately after they were thawed and cultured.

\section{Cell preparation for flow cytometry (FCM) and cell counting}

The cells were fixed with $20 \%$ ethanol, and then incubated with $0.25 \%$ RNase (Type II-A, Sigma, St. Louis, MO, USA) for $3 \mathrm{~h}$ at $4^{\circ} \mathrm{C}$. The cells were counted using a hemocytometer. Immediately before the measurements, the cells were stained with propidium iodide (PI, $7.5 \times 10^{-5} \mathrm{M}$ ) and examined for red fluorescence by FCM. Under these staining conditions, the signal due to residual double-stranded RNA is negligible and the relative intensity of the red fluorescence corresponds to the DNA content (Krishan 1975).

\section{Flow cytometry (FCM)}

The fluorescence from individual cells was measured using a FACSORT (Becton Dickinson Immunocytometry Systems, Franklin Lake, ND, USA). The fluorescence of individual cells irradiated with a focused laser light at a wavelength of $488 \mathrm{~nm}$ was detected using a photomultiplier tube. The relative intensity of red fluorescence (FL2A) was measured and DNA histograms were obtained. The coefficient of variation $(\mathrm{CV})$ of DNA histograms was obtained using computer software (ModFit, Becton Dickinson Immunocytometry Systems).

\section{Chromosome number}

Exponentially growing diploid, tetraploid and octaploid Meth-A cells were plated in culture dishes ( $60 \mathrm{~mm}$ diameter, Nalge Nunc International, IL, USA) at a density of about $5 \times 10^{5}$ cells/dish. Twelve hours thereafter, the cells were exposed to demecolcine at a concentration of $270 \mathrm{nM}$ for $1 \mathrm{~h}$. The cells were swelled by $75 \mathrm{mM} \mathrm{KCl}$, fixed with a fixing solution $\left(\mathrm{CH}_{3} \mathrm{OH}: \mathrm{CH}_{3} \mathrm{COOH}=7: 3\right)$ and dropped onto glass slides. The cells were stained with Giemsa solution and then the chromosomes were photographed. The chromosome number was counted from the photographs and histograms of the chromosome number (chromosome distribution) were obtained. 


\section{Cell morphology}

The morphology of exponentially growing diploid, tetraploid and octaploid Meth-A cells in culture flasks was photographed under a low-phase contrast microscope (CK2, Olympus, Tokyo, Japan) equipped with a digital camera system (DS4040, Olympus). The images were entered into a personal computer and printed out at the magnification stated in the text.

\section{Simulation curves}

The simulation curves of $\mathrm{f}(\mathrm{t})$ were obtained using computer software (Mathematica, Wolfram Research Inc.).

\section{Results}

To examine DNA loss in polyploid cells, diploid, tetraploid and octaploid Meth-A cells were continuously cultured for $244 \mathrm{~d}$. Representative DNA histograms of the cells are shown in Fig. 1. At $0 \mathrm{~d}$, the $\mathrm{G}_{1}$ peaks of tetraploid and octaploid Meth-A cells were positioned near the $\mathrm{G}_{2}$ peaks of diploid and tetraploid Meth-A cells, respectively, meaning that these cells had normal DNA content. At $240 \mathrm{~d}$, the positions of the $\mathrm{G}_{1}$ peaks of tetraploid and octaploid Meth-A cells were shifted to the late $\mathrm{S}$ phase regions of diploid and tetraploid cells, respectively, suggesting that DNA had been lost in the polyploid cells.

To examine the changes in DNA content of polyploid Meth-A cells, the positions of the $G_{1}$ peaks of tetraploid and octaploid Meth-A cells relative to those of diploid cells were plotted against the days in culture (Fig. 2). Because the electric origin of DNA histograms may drift from measurement to measurement, the positions of the $G_{1}$ and $G_{2} / M$ peaks of diploid cells were regarded as

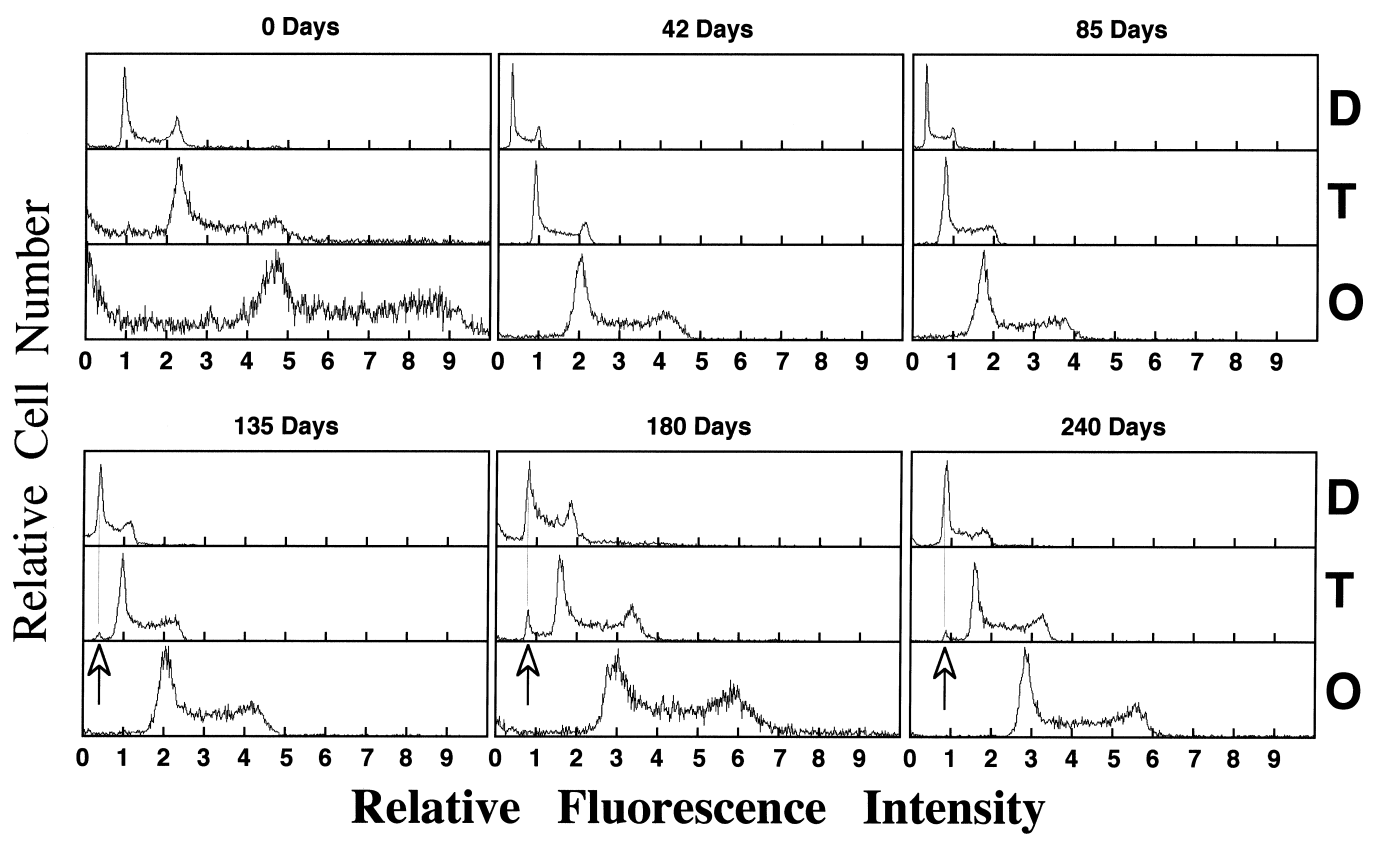

Fig. 1. Changes in DNA fluorescence histograms of diploid (D), tetraploid (T) and octaploid (O) Meth-A cells during $240 \mathrm{~d}$ of culture. Tetraploid and octaploid Meth-A cells stored in the early stage of establishment were re-cultured from freezing. The measurements were made starting $3 \mathrm{~d}$ after the reculturing. The diploid, tetraploid and octaploid Meth-A cells were subcultured almost every day in culture flasks. The abscissa represents the relative intensity of red fluorescence. The histograms at a day were obtained under the same FCM conditions. Arrows in 135, 180 and $240 \mathrm{~d}$ indicate peaks newly appeared. 


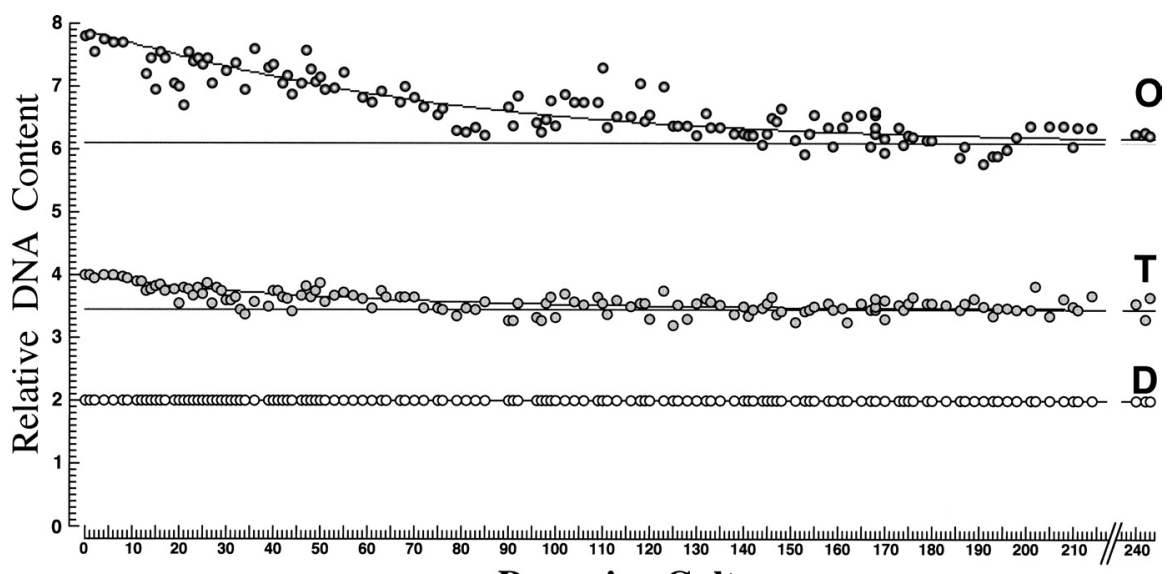

\section{Days in Culture}

Fig. 2. Time-course of relative DNA content of octaploid (O), tetraploid (T) and diploid (D) Meth-A cells. The DNA content of tetraploid and octaploid cells was determined from the $G_{1}$ peak position in comparison with that of diploid cells. The abscissa represents days in culture. The solid lines represent the experimental equations: $y=7.9 \exp [-0.026 \mathrm{t} /\{\exp (-0.01 \mathrm{t})+0.01 \mathrm{t}\}]$ for octaploid cells, $y=4 \exp [-0.026 \mathrm{t} /\{\exp (-0.01 \mathrm{t})+0.0175 \mathrm{t}\}]$ for tetraploid cells and $\mathrm{y}=2$ for diploid cells, where $\mathrm{t}$ is days in culture. The dotted lines $(y=6.091$ for octaploid and $y=3.447$ for tetraploid) were drawn to facilitate understanding of the terminal DNA content calculated using the equation.

those corresponding to $2 \mathrm{C}$ and 4C DNA content, respectively, where $\mathrm{C}$ means the complement of haploid chromosomes. It should be emphasized that the peak position is shifted by many experimental conditions, such as the cell density, the PI concentration in the sample solution, the temperature of the sample solution, the flow rate of the sheath fluid, the DNA content of cells, the $\mathrm{pH}$ of the sample solution and the optical and electrical conditions of the FCM machine. Although we measured DNA histograms at the same time with the same electrical conditions of the FCM machine, the resultant peak positions were fairly shifted each day, and the plots were considerably dispersed.

The DNA content of tetraploid and octaploid Meth-A cells decreased rapidly in the early stage of culture, and then reached a plateau where the DNA level was higher than that of diploid cells. The DNA content at time $\mathrm{t}$ (days), $\mathrm{f}(\mathrm{t})$, could be described as $\mathrm{f}(\mathrm{t})=\mathrm{I}_{\mathrm{p}} \exp \{-\mathrm{G}(\mathrm{t})\}$, where $\mathrm{I}_{\mathrm{p}}=4$ (for tetraploid cells) or $=7.9$ (for octaploid cells), $G(t)=a t /\{\exp (-b t)+c t\}$, and $a, b$ and $c$ are the following parameters: $a=0.026, b=0.01$ and $c=0.0175$ (for tetraploid cells) or $c=0.01$ (for octaploid cells). Diploidy was clearly not the final ploidy. The meaning of the parameters $a, b$, and $c$ will be discussed in the Discussion.

To examine whether or not diploid cells lost DNA, diploid Meth-A cells stored at $-135^{\circ} \mathrm{C}$ were thawed and placed in culture and their DNA histograms were compared with those of diploid Meth-A cells that had been cultured for over $240 \mathrm{~d}$ (Fig. 3). Though the $\mathrm{G}_{1}$ peak position of Meth-A cells that had been cultured for over $240 \mathrm{~d}$ was slightly shifted right compared to that of the control, the amount of DNA loss was within experimental error. It was concluded that the DNA content of diploid Meth-A cells could be regarded as a constant within experimental error.

To confirm that tetraploid and octaploid Meth-A cells lost chromosomes during cell culturing, the chromosome number was counted. Figure 4 shows the distribution of chromosome number for diploid, tetraploid and octaploid Meth-A cells. The modes of the chromosome number in the longterm cultured tetraploid and octaploid Meth-A cells were 78 and 127, respectively, and were smaller than those in the control cultures ( 88 and 158, respectively), suggesting that the cells lost a part of the chromosomes, or that the chromosomes combined with each other.

To examine whether or not the cellular morphology was altered in the long-term culture, the morphology of growing cells was compared at 0 and $>240 \mathrm{~d}$ (Fig. 5). A distinct alteration in cell 
morphology was observed in diploid and octaploid Meth-A cells, but not in the tetraploid cells. The morphology of diploid and octaploid Meth-A cells changed from a suspended sphere-like shape to a monolayered amoebalike shape. The suspended, sphere-like and cell-cell aggregating characteristics of tetraploid Meth-A cells did not change throughout the cell culturing. Subtle distinctions among the culture flasks were also observed in the octaploid Meth-A cells. The morphology alteration that arose during long-term culture was clearly independent of the DNA loss, because the alteration occurred in diploid cells, whose loss of DNA content was negligibly small.

To examine whether or not the cell proliferation was altered in the long-term culture, the doubling time was measured by determining the cell-counts in subcultures of diploid, tetraploid and octaploid Meth-A (Table 1). The doubling time of diploid cells was not altered

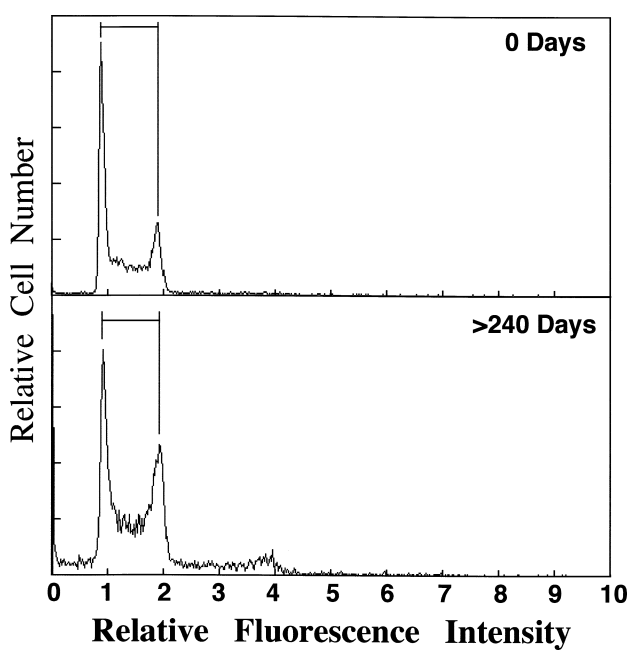

Fig. 3. The DNA fluorescence histograms of diploid Meth-A cells at 0 (upper) and over 240 (lower) days of culture. Diploid Meth-A cells stored at $-135^{\circ} \mathrm{C}$ were recultured and used as the $0 \mathrm{~d}$ sample. The measurements were made using the same FCM gain. The abscissa represents the relative intensity of red fluorescence.
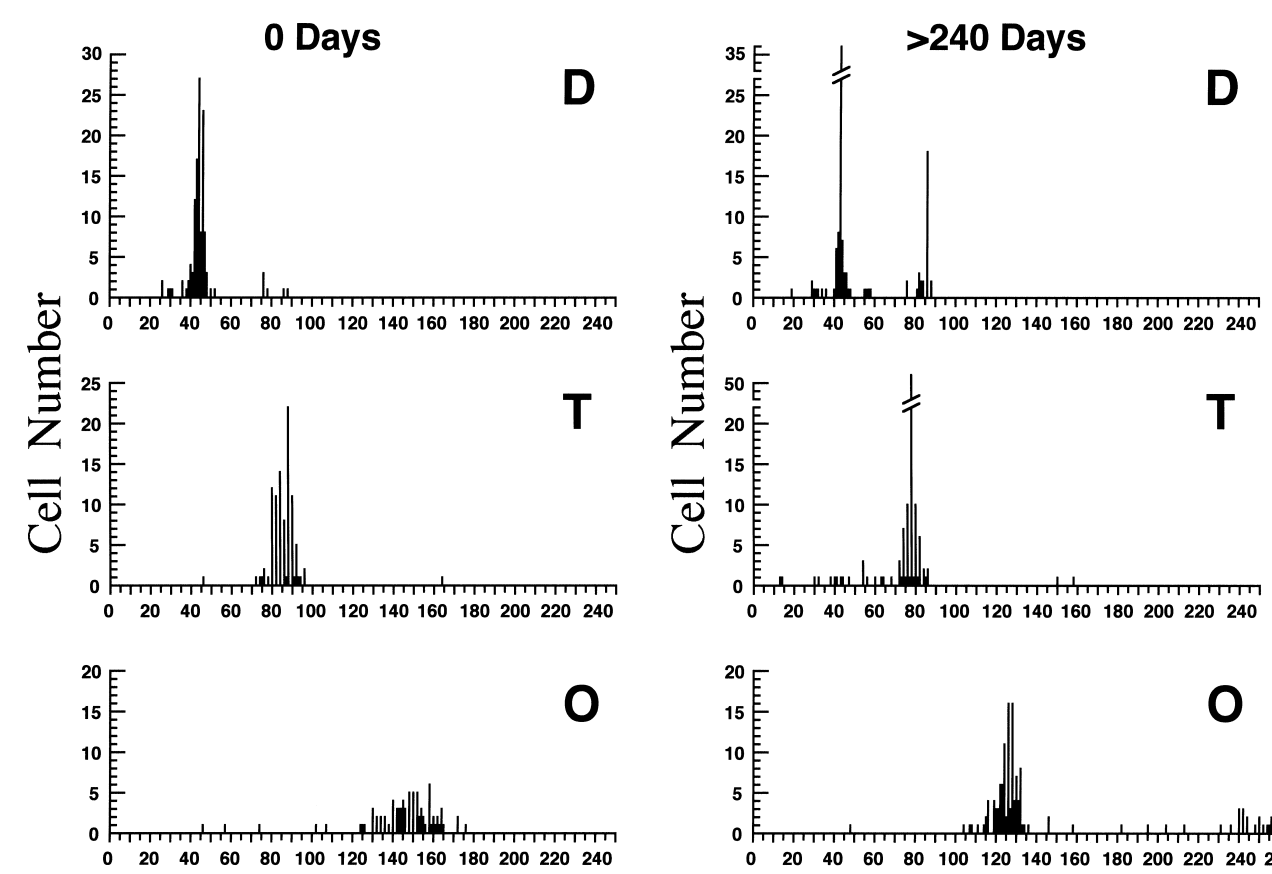

Chromosome Number

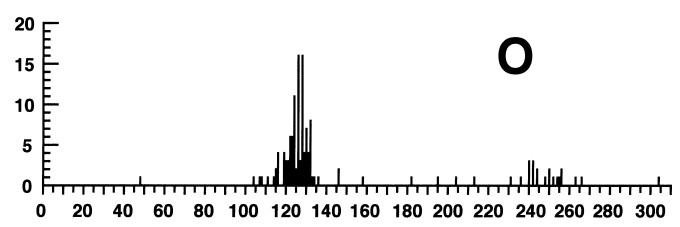

Chromosome Number

Fig. 4. Histograms of chromosome number of diploid (D), tetraploid (T) and octaploid (O) Meth-A cells at 0 days (left panels) and in over $240 \mathrm{~d}$ (right panels) of culture. Exponentially growing diploid, tetraploid and octaploid Meth-A cells were exposed to $270 \mathrm{nM}$ demecolcine for $1 \mathrm{~h}$. The chromosomes were stained with Giemsa solution. The chromosomes of about 100 cells were enumerated from enlarged photographs. 

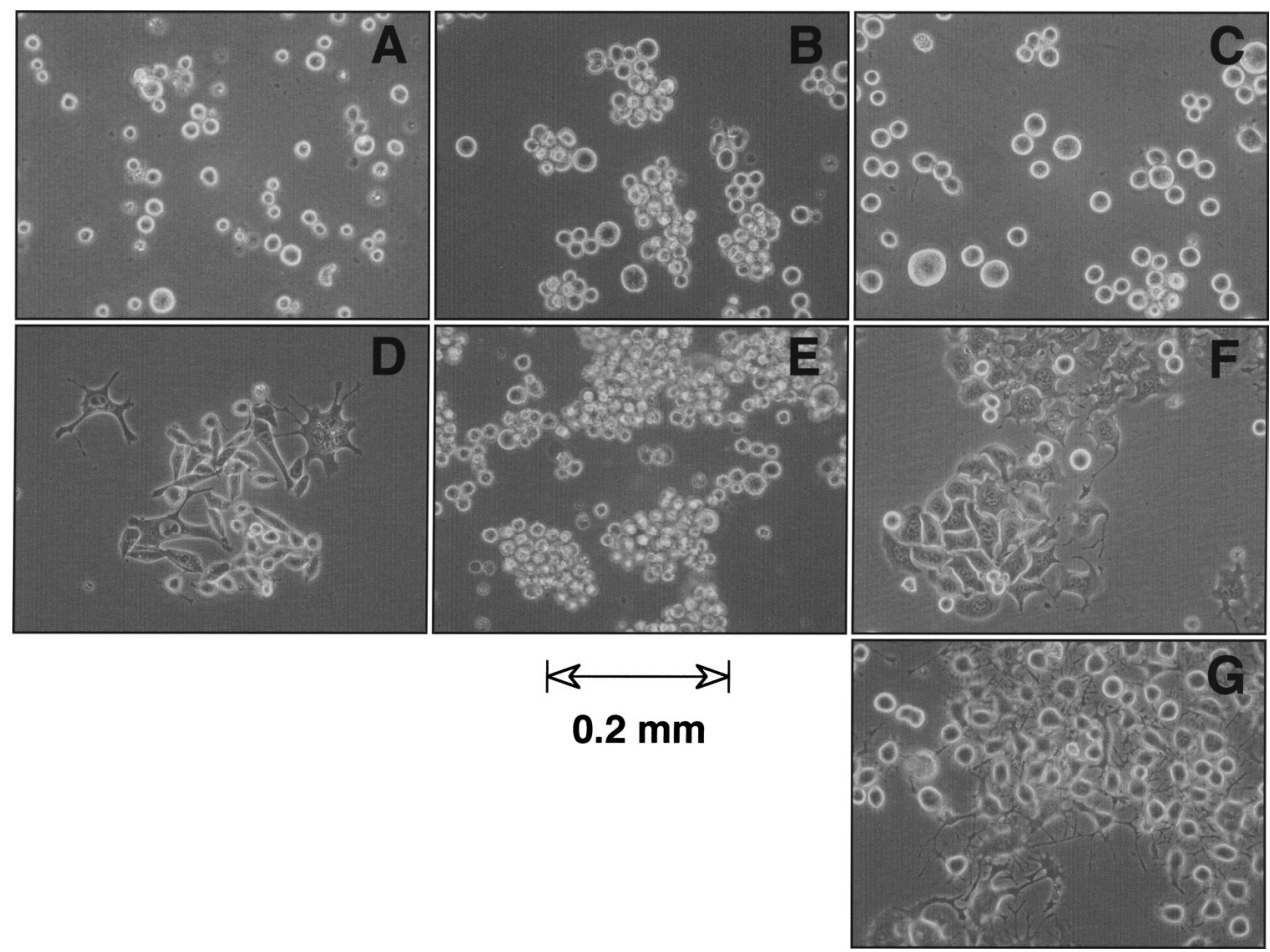

Fig. 5. Phase-contrast micrographs of exponentially growing diploid (A and D), tetraploid (B and E) and octaploid (C, F and G) Meth-A cells at $0 \mathrm{~d}$ (A, B and C) and over $240 \mathrm{~d}$ (D, E, F and G) of culture. The photographs in $\mathrm{F}$ and $\mathrm{G}$ are from different flasks.

Table 1. Changes in doubling times (h) of diploid, tetraploid and octaploid Meth-A cells*

\begin{tabular}{lccccc}
\hline \hline Period (d) & $0-20$ & $35-55$ & $80-100$ & $125-145$ & $160-180$ \\
\hline Diploid & 22.0 & 22.8 & 22.2 & 21.4 & 22.0 \\
Tetraploid & 25.2 & 24.0 & 23.5 & 20.4 & 20.4 \\
Octaploid & 28.8 & 26.4 & 25.5 & 23.4 & 21.6 \\
\hline
\end{tabular}

* The doubling times were calculated from changes in the cell number of cell populations. Note that the terms "tetraploid" and "octaploid" have been used irrespectively of the DNA content of cell cultures.

within the experimental error, whereas those of tetraploid and octaploid cells decreased. The decrease in the doubling time, however, may not have been related to the DNA loss in the polyploid cells, because the doubling time was shorter than that of diploid cells.

\section{Discussion}

The DNA content of tetraploid and octaploid Meth-A cells at time $t$ (day), $f(t)$, could be described within experimental error as $\mathrm{f}(\mathrm{t})=\mathrm{I}_{\mathrm{p}} \exp \{-\mathrm{G}(\mathrm{t})\}$, where $\mathrm{I}_{\mathrm{p}}=4$ (for tetraploid cells) or $=7.9$ (for octaploid cells), $\mathrm{G}(\mathrm{t})=\mathrm{at} /\{\exp (-\mathrm{bt})+\mathrm{ct}\}$, and $\mathrm{a}, \mathrm{b}$ and $\mathrm{c}$ are the following parameters: $a=0.026, b=0.01$ and $c=0.0175$ (for tetraploid cells) or $c=0.01$ (for octaploid cells). $I_{p}$ is the initial ploidy, since $\mathrm{f}(0)=\mathrm{I}_{\mathrm{p}}$. The rate of DNA reduction, $-\mathrm{df}(\mathrm{t}) / \mathrm{dt}$, is a $\mathrm{I}_{\mathrm{p}}$ at $\mathrm{t}=0$ and 0 at infinity, irrespective of $b$ and $c$. The parameter a may represent the probability of DNA loss in the polyploid Meth-A cells at the start of the culture.

In order to examine the characteristics of these parameters, simulation curves of $f(t)$ were 
drawn by varying the parameters (Fig. 6). With the decrease of parameter a, the decay rate increases. As parameter $b$ increases, the decay becomes more rapid in the early stage of culture and becomes more sigmoidal. When parameter $\mathrm{c}$ increases, the terminal ploidy decreases. At present, the meaning of these parameters is not clear. Furthermore, more suitable functions might be found. The value of parameter $\mathrm{a}=0.026$, however, suggests that octaploid and tetraploid Meth-A cells, having about 160 and 80 chromosomes, will lose 4 and 2 chromosomes per cell division in the early stage of culture, respectively.

One interesting question is whether specific chromosomes were destined to be lost. To examine whether or not the polyploid cells lost the same kinds of chromosomes in a cell population, the CV values of DNA histograms were calculated. Although CV values of DNA histograms will be affected by many experimental conditions both of the samples and the FCM machine, they are the only available indicator of homogeneity of cellular DNA content (ploidy) in a cell population. We detected small CV values during the culture period examined (Fig. 7). The CV values for tetraploid and octaploid Meth-A cells increased gradually until about $180 \mathrm{~d}$ (from 4 to $5.2 \%$ (tetraploid cells) and 7.0\% (octaploid cells)) and then regressed to the initial level by $240 \mathrm{~d}$. In contrast, the $\mathrm{CV}$ values for diploid Meth-A cells did not change with time. It seems that the DNA homogeneity of populations of tetraploid and octaploid Meth-A cells decreas-
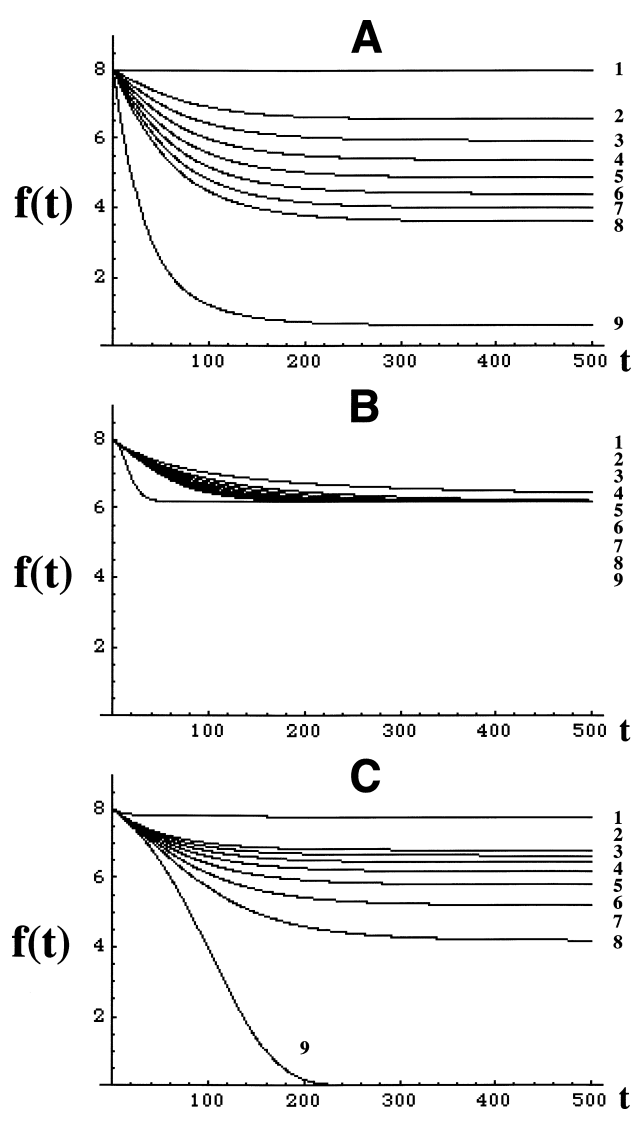

Fig. 6. Simulation curves of $f(t)=8.0 \exp [-a t /\{\exp (-$ $\mathrm{bt})+\mathrm{ct}\}$ ] changing a (A), b (B) and $\mathrm{c}(\mathrm{C})$. The fixed values of the parameters are $a=0.026$, $\mathrm{b}=0.01$ and $\mathrm{c}=0.01$. The abscissa represents $\mathrm{t}$. In $\mathrm{A}$, parameter a is changed as 0.0 (1), 0.002 (2), 0.003 (3), 0.004 (4), 0.005 (5), 0.006 (6), 0.007 (7), 0.008 (8) and 0.026 (9). In B and C, parameters $\mathrm{b}$ and $\mathrm{c}$ are respectively varied as 0.0 (1), 0.004 (2), 0.006 (3), 0.008 (4), 0.01 (5), $0.012(6), 0.014(7), 0.016(8)$ and 0.1 (9). es with culture time. In the early stage of culture, however, the CV values retained near the initial level, suggesting that the same amount of DNA was lost in most cells in the population. It is probable that the chromosomes to be lost are previously determined in each cell in a population. Unfortunately, we could not determine what kinds of chromosomes were lost at first, because the karyotype of Meth-A cells is not stable like the karyotypes of many mammalian cell lines.

It is interesting that a clear $\mathrm{G}_{1}$ peak of intact diploid cells appeared in the DNA histograms at 135,180 and $240 \mathrm{~d}$ in a population of cells whose ploidy was about 3.5 (indicated by arrows in Fig. 1). No sub- $\mathrm{G}_{1}$ peaks were observed in the octaploid cell population, at least for $240 \mathrm{~d}$ (data not shown). These phenomena mean that a hypotetraploid-diploid transition (reverse transformation) occurred in hypotetraploid Meth-A cells and that an intact set of diploid chromosomes is preserved in the process of ploidy degradation of tetraploid Meth-A cells, but not of octaploid cells. From these experimental results, one can make the important prediction that chromosomes are not independent in polyploid cells, but are present in units of the diploid set. A model that has been proposed for the triploid V79 cells may be applicable (Fujikawa-Yamamoto et al. 2002). 


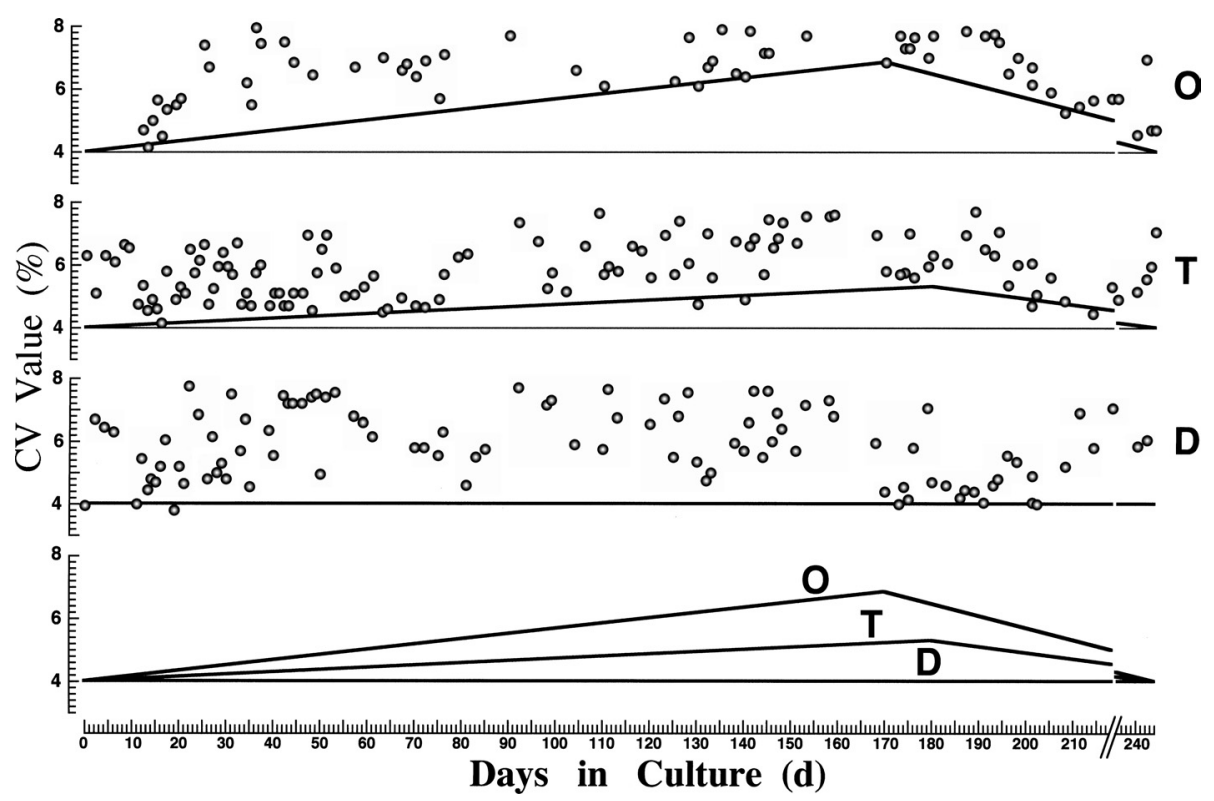

Fig. 7. The coefficient of variation (CV) of DNA histograms of diploid (D), tetraploid (T) and octaploid (O) Meth-A cells during $244 \mathrm{~d}$ of continuous culture. The CV values were obtained using computer software (ModFit). The values above $8 \%$ were cut off. Solid lines were drawn to facilitate understanding.

It should be emphasized that the present results about DNA loss apply only to variation in a long-term culture of a particular type of proliferating cells, Meth-A cells.

\section{References}

Fujikawa-Yamamoto, K., Zong, Z., Murakami, M., Odashima, S., Ikeda, T. and Yoshitake, Y. 1997. Spontaneous polyploidization results in apoptosis in a Meth-A tumor cell line. Cell Struct. Funct. 22: 399-405.

-, Wang, S., Yamagishi, H., Ohdoi, C., Murano, H. and Ikeda, T. 2001. Establishment of a tetraploid Meth-A cell line through polyploidization by demecolcine but not by staurosporine, K-252a and paclitaxel. Cell Prolif. 34: 211-222.

- Yamagishi, H. and Miyagoshi, M. 2002. Establishment of a triploid V79 cell line from tetraploid cells obtained through polyploidization using K-252a. Cell Prolif. 35: 369-379.

—, - and - 2003. Octaploid Meth-A cells are established from a highly polyploidized cell population. Cell Prolif. 36: $87-100$.

-, Sakuma, M. 2003. Maintenance of quantity and density of membrane glycochains in diploid-tetraploid and tetraploidoctaploid transitions, respectively of Meth-A cells demonstrated by lectin binding. Cytologia 68: 191-198.

Graves J. A. M. and McMillan, J. 1984. Control of DNA synthesis in polyploid mammalian cells. J. Cell. Physiol. 121: 409-414.

Harris, M. 1971. Polyploid series of mammalian cells. Exp. Cell Res. 66: 329-336.

Krishan, A. 1975. Rapid flow cytofluorometric analysis of mammalian cell cycle by propidium iodide staining. J. Cell Biol. 66: $188-193$.

Moore, J., Kieler, J. and Biczowa, B. 1968. Comparative studies of a near-tetraploid and a near-diploid line of Ehrlich's ascites tumor propagated in vivo and in vitro II. Cytology and transplantability. Eur. J. Cancer 4: 81-95. 